\title{
ANSCHRIFTEN DER AUTOREN
}

Gesandter Dr. Rudolf Agstner

Bundesministerium für auswärtige Angelegenheiten

Ballhausplatz 2

A-1010 Wien

E-mail: rudolf.agstner@bmaa.gv.at

Dr. Micaela Antonucci

Via dei Panfili, 105

I-00121 Roma

E-mail: micaela_antonucci@fastweb.it

Dr. Angela Dreszen

Bibliotheca Hertziana (Max-Planck-Institut)

Via Gregoriana, 28

I-00187 Roma

E-mail: dressen@biblhertz.it

Dr. Krystyna Frącz

Sagbergstr. 69 A

A-3011 Neu-Purkersdorf

E-mail:kika@newsclub.at

Dr. Maddalena GuiotTo

Istituto storico italo-germanico in Trento

Via S. Croce, 77

I-38100 Trento

E-mail:mguiotto@libero.it

Mag. Dr. Julia Hörmanx

Institut für Geschichte

Universität Innsbruck

Innrain 52

A-6020 Innsbruck

E-mail: julia.hoermann@uibk.ac.at

Dr. Varduì KalPaKcian

Corso Vittorio Emanuele II, 46

I-35123 Padova

E-mail: hovannes@libero.it 
Mag. Thomas Kuster

Josef Schrafflstr. 1

A-6020 Innsbruck

E-mail: thomas_kuster@hotmail.com

Dr. Claudia Lamberti

Via Napoli, 25

I-56123 Pisa

E-mail: c.lamberti@ing.unipi.it

Dr. Rainer Murauer

Historisches Institut beim Österr. Kulturforum in Rom

Viale Bruno Buozzi, 113

I-00197 Roma

E-mail: r.murauer@tin.it

Dr. Luigi Pedroni

Dr. Stumpf-Str. 117

A-6020 Innsbruck

E-mail: luipedro@tin.it

Dr. Georg A. Plattiner

Österreichisches Archäologisches Institut

Franz Klein-G. 1

A-1190 Wien

E-mail: georg.plattner@oeai.at

PD Dr. Ulrike SeEger

Im Degen 46

D-70327 Stuttgart

E-mail: ulrikeseeger@web.de

Mag. Dr. Pia Theis

Universität Wien

Institut für Kunstgeschichte

Spitalgasse 2-4, Hof 9

A-1090 Wien

E-mail: pia.theis@univie.ac.at

Dr. Antonio Trampus

Dipartimento di Studi Storici

Università Ca' Foscari Venezia

Ca' Garzoni-Moro

San Marco. 3417

I-30124 Venezia

E-mail: trampus@unive.it 

